\title{
Feridas em pacientes diabéticos
}

\author{
Wounds in diabetic patients
}

\section{Viviane Fernandes Carvalho', Pedro Soler Coltro², Marcus Castro Ferreira ${ }^{3}$}

\author{
Carvalho VF, Coltro OS, Ferreira MC. Feridas em pacientes diabéticos. Rev Med (São Paulo). 2010 \\ jul.-dez.;89(3/4):164-9.
}

\begin{abstract}
RESUMO: No Brasil, a prevalência do diabetes na população brasileira acima de 40 anos de idade é de 10\%, com estimativa de mais de 3,6 milhões de usuários do sistema público. Entre as complicações sérias e onerosas que afetam os pacientes diabéticos, aquelas que ocorrem nos membros inferiores (MMII) representam a maior parte delas (40 a 70\%). A microangiopatia atinge os pequenos vasos responsáveis pela irrigação nervosa, os chamados vasa nervorum e é. a desencadeadora da neuropatia periférica, retinopatia e nefropatia diabética. $O$ processo neuropático, pelo fato de favorecer deformidades ósseas no pé, acarreta aumento das pressões plantares. A associação da insensibilidade do pé e do aumento destas pressões resulta em ulceração. Desde 1988, com o desenvolvimento do Pressure Specified Sensory Device ${ }^{\mathrm{TM}}$ - PSSD, um só equipamento é capaz de quantificar o limiar de pressão aplicada sobre a pele, necessário para que o paciente sinta o estímulo de um ponto estático e um ponto dinâmico assim como os testes com dois pontos. Consegue-se melhor avaliação do déficit sensitivo nos pés e melhor planejamento de medidas terapêuticas. 0 tratamento da ferida diabética envolve o controle da isquemia, se necessário e de desbridamento cirúrgico. O sistema a vácuo auxilia na redução do edema, na melhora do aporte sanguíneo ao leito e na formação do tecido de granulação, acelerando o preparo do leito e tornando mais precoce a indicação do tratamento cirúrgico definitivo, através em geral com uso de enxertos de pele.
\end{abstract}

DESCRITORES: Ferimentos e lesões/complicações; Pé diabético/complicações; Desbridamento/ métodos; Úlcera por pressão; Cirurgia plástica.

1. Enfermeira-Doutora da Divisão de Cirurgia Plástica e Queimaduras do Hospital das Clínicas da da Faculdade de Medicina da Universidade de São Paulo (HCFMUSP).

2. Médico Assistente da Divisão de Cirurgia Plástica e Queimaduras do Hospital das Clínicas da da Faculdade de Medicina da Universidade de São Paulo (HCFMUSP).

3. Professor Titular da Disciplina de Cirurgia Plástica da da Faculdade de Medicina da Universidade de São Paulo (FMUSP). Endereço para correspondência: Viviane Fernandes Carvalho. Laboratório de Investigação Médica (LIM 04). Av. Dr. Arnaldo, 455 - SI. 1363 - São Paulo, SP, Brasil. CEP: 01246-903. 
nteriormente à descoberta da insulina
exógena, os pacientes portadores
de diabetes melitus (DM) morriam devido às alterações sistêmicas da doença, como a hiperglicemia e a cetoacidose. Com o advento da insulinoterapia e dos hipoglicemiantes orais, estes indivíduos passaram a ter maior expectativa de vida, e as complicações, na maioria das vezes, resultam do estado hiperglicêmico crônico. As complicações são divididas em dois grandes grupos: vasculopatia e neuropatia. As vasculopatias podem ser divididas em dois subgrupos: a macroangiopatia, responsável pela doença cardiovascular e cerebrovascular e a microangiopatia, desencadeadora da neuropatia periférica, retinopatia e nefropatia diabética ${ }^{1,2,3}$.

No Brasil, a prevalência do diabetes na população brasileira acima de 40 anos de idade é de $10 \%$, com estimativa de mais de 3,6 milhões de usuários do sistema público; quase metade desconhecia esse diagnóstico e somente 2/3 estão em seguimento em unidades básicas de saúde.

Embora muitas sejam as complicações sérias e onerosas que afetam os pacientes diabéticos, aquelas que ocorrem nos membros inferiores (MMII) representam a maior parte delas (40 a 70\%). A consequência mais grave do diabetes melitus nos MMII é a amputação, parcial ou total, de um ou ambos os membros, ou ainda a formação de feridas de difícil resolução4.

A causa mais comum (60 a $70 \%$ dos casos) da ulceração e da amputação é a perda da sensibilidade cutânea protetora, resultante da neuropatia. Esta perda pode ser encontrada em até $10 \%$ dos pacientes quando o DM é diagnosticado e em cerca de $50 \%$ dos indivíduos diabéticos com mais de 20 anos de evolução da doença ${ }^{5}$.

A neuropatia atinge nervos sensitivos, motores e autônomos. O funcionamento anormal dos nervos sensitivos acarreta perda da sensibilidade protetora, dor e dormência nos membros inferiores. A neuropatia motora pode resultar em atrofia dos músculos. Isso gera desequilíbrio entre os grupos musculares, levando a deformidades do pé. Quando os nervos autônomos são atingidos, há diminuição da função sudomotora e o fluxo sangüíneo encontrase aumentado nas solas dos pés ${ }^{6}$. No diabético, o pé é mais vulnerável, pois a neuropatia favorece o aparecimento de feridas, as quais tendem à infecção, colocando em risco a viabilidade do membro afetado e a vida do paciente.

Para entendermos a formação das úlceras diabéticas, devemos compreender a fisiologia do pé. As principais funções do pé são sustentação do corpo, absorção das forças de impacto, ajuste às superfícies irregulares, proporcionar alavanca para propulsão e proteção de tecidos moles. Os fatores que alteram a distribuição normal das forças de locomoção, irrigação ou sensibilidade do pé, como ocorrem nos diabéticos, potencializam o risco de lesão no local ${ }^{7}$.

Nos diabéticos, podemos encontrar alterações nos troncos arteriais ou arteríolas e capilares, caracterizando, respectivamente, a macroangiopatia e microangiopatia.

O aparecimento de aterosclerose está acelerado no DM, elevando as taxas de morbimortalidade ${ }^{8}$. Praticamente todos os grandes vasos (macroangiopatia) estão envolvidos nesse processo e as manifestações clínicas aparecem em conseqüência de estreitamento aterosclerótico e de trombose dos vasos coronarianos, cerebrais e das pernas. Nos MMII, há predomínio do processo degenerativo nos vasos infra-inguinais, com ênfase nas artérias tibial e fibular.

A microangiopatia é característica do DM. Nas arteríolas, observamos hipertrofia e proliferação da camada íntima e nos capilares há espessamento da membrana basal $(\mathrm{MB})^{9}$. Devido a estas alterações estruturais, a permeabilidade vascular é aumentada e a auto-regulação do tônus vascular fica comprometida.

A etiologia da neuropatia periférica é dupla, vascular e metabólica. A neuropatia é conseqüência da microangiopatia, uma vez que esta atinge os pequenos vasos responsáveis pela irrigação nervosa, os chamados vasa nervorum. A resistência vascular aumentada na vasa nervorum diminui a irrigação endoneural, causando hipóxia e menor atividade da bomba $\mathrm{Na}^{+} / \mathrm{K}^{+}$ATPase, com consequente queda na velocidade de condução de impulsos nervosos.

Todavia a diminuição da velocidade de condução nervosa mediada pela atividade da bomba $\mathrm{Na}+/ \mathrm{K}+$ ATPase, também está relacionada às alterações metabólicas. As altas concentrações de glicose no meio intracelular, em ambiente hiperglicêmico e hipoinsulinêmico, são convertidas em sorbitol pela enzima aldose redutase ${ }^{10}$. Esta via, conhecida como via do poliol, fica inativada em situações de normoglicemia.

O processo neuropático, pelo fato de favorecer deformidades ósseas no pé, acarreta aumento das pressões plantares. A associação da insensibilidade do pé e do aumento destas pressões resulta em ulceração.

Ocasionalmente, os pés de pacientes com neuropatia são afetados por desorganização óssea através do aparecimento da neuroartropatia de Charcot. $\mathrm{O}$ pé de Charcot é um processo destrutivo não infeccioso dos ossos e das articulações, causado pela fratura e/ou luxação associados à neuropatia 
periférica $^{11,12}$.

A prevalência global de amputações é 10 vezes maior em pacientes diabéticos do que em indivíduos sem a doença $(2,8 \% \times 0,29 \%)^{13}$. Sobre a incidência de amputações não relacionadas a traumatismos nos MMII, os índices variam de 2,1 por 1.000 a 13,7 por $1.000^{14}$, demonstrados em estudos com 14 anos de evolução. A incidência por ano de feridas em MMII de diabéticos varia de 1,0 a 4,1\% ${ }^{15}$, enquanto que a prevalência destas lesões oscila entre 5,3 e $10,5 \%$.

Os fatores de risco para formação de feridas e amputação nos MMII são o tempo de evolução do $\mathrm{DM}^{9,16,17}$, a doença vascular periférica ${ }^{18}$, o nível de hemoglobina glicosilada $\left(\mathrm{HbA}_{1 \mathrm{c}}\right)^{17}$, a presença de deformidade óssea, a história de ferida prévia e de amputação pregressa. A neuropatia periférica representa um processo insidioso e progressivo para as mais graves complicações nos MMII, inerentes ao $\mathrm{DM}^{19,20}$. A neuropatia diabética é definida como uma lesão do nervo periférico, somático ou autônomo, atribuível exclusivamente ao DM. Está presente, em algum grau, em mais de $50 \%$ dos diabéticos com mais de 60 anos de idade.

Os nervos periféricos são classificados em autônomos, motores e sensitivos e a lesão provocada pelo DM altera a fisiologia desses três grupos de nervos. A neuropatia autônoma pode levar à diminuição da função sudomotora. Lesões dos nervos motores acarretam atrofias musculares. Já a neuropatia sensorial resulta em alterações como parestesia, hipoestesia e perda da sensibilidade protetora, sendo um processo irreversível.

A diminuição ou perda da sensibilidade protetora torna o diabético mais vulnerável ao trauma físico e térmico, conseqüentemente levando-o à formação de feridas e eventualmente a perda segmentar nos MMII. Há técnicas cirúrgicas e procedimentos clínicos para se evitar a progressão dos danos provocados pela neuropatia diabética, entretanto não há consenso sobre qual seria o melhor tratamento ${ }^{21}$. A maioria dos especialistas indica medidas de prevenção, como a melhor forma de se conter as consequências impostas pela neuropatia diabética ${ }^{22,23,24}$.

A profilaxia das complicações neuropáticas tem início com a identificação do grau de neuropatia e, portanto, do déficit neurológico apresentado pelos pacientes diabéticos. Isto é possível através de testes de avaliação de sensibilidade cutânea nos MMII. Os principais testes disponíveis para examinar a perda funcional de sensibilidade cutânea estão relacionados à sensação tátil, à pressão e à vibração. Esta qualidade de sensação é mediada através dos axônios mielinizados, conhecidos como fibras de adaptação rápida e de adaptação lenta, conforme seu receptor periférico ${ }^{25}$.

Em 1962, Weinstein ${ }^{26}$ adaptou monofilamentos de nylon em hastes plásticas, método conhecido atualmente como monofilamento de SemmesWeinstein ${ }^{\circledR}$, tido como o "Gold Standard" das medidas de sensibilidade nos diabéticos, devido a sua simplicidade, rapidez e baixo custo do teste ${ }^{26}$. O monofilamento é aplicado perpendicularmente à superfície do corpo e a pressão é aumentada até que o filamento forme um arco. Este teste quantifica a pressão necessária para percepção do tato e avalia a função do conjunto fibra/receptor de adaptação lenta.

Para avaliar a função das fibras de adaptação rápida e seus receptores periféricos são feitas manobras com instrumentos produtores de vibração, por exemplo, diapasões ${ }^{27}$. Associando o exame de sensibilidade vibratória, realizado com o diapasão de $128 \mathrm{~Hz}$, com a avaliação de sensibilidade pressórica, dada pelo monofilamento de Semmes-Weinstein ${ }^{\circledR}$, a sensibilidade do teste foi de $100 \%$ e a especificidade de $77 \%$ para evolução da neuropatia diabética.

Desde 1988, com o desenvolvimento do Pressure Specified Sensory Device ${ }^{\mathrm{TM}}$ - PSSD, um só equipamento é capaz de quantificar o limiar de pressão aplicada sobre a pele, necessário para que o paciente sinta o estímulo de um ponto estático (semelhante ao monofilamento de SemmesWeinstein ${ }^{\circledR}$ ), um ponto em movimento (semelhante a diapasão), dois pontos estáticos e dois pontos em movimento. Temos, portanto, um meio direto para se avaliar os sistemas de fibras de adaptação lenta e rápida e seus respectivos receptores periféricos.

O tratamento das feridas nos pés de pacientes diabéticos deve adotar uma abordagem multidisciplinar para o controle do DM e das complicações associadas. A obtenção do controle glicêmico é importante, além da interrupção do tabagismo, do cuidado com os pés e do uso de calçados apropriados. Em todo esse processo, a educação do paciente sobre a doença e sobre os cuidados é fundamental.

O tratamento da ferida diabética envolve o controle da isquemia, com redução do risco cardiovascular. Em alguns pacientes, pode ser necessário a revascularização dos MMII. A ferida deve ser limpa e o material necrótico deve ser desbridado. Se houver sinais de infecção, o tratamento deve ser complementado com antibióticos, de acordo com a cultura da ferida. Nos casos de osteomielite, a terapia antimicrobiana demanda um tempo maior e requer, frequentemente, ressecções cirúrgicas mais amplas.

O leito da ferida deve ser preparado para 
promover os mecanismos de cicatrização, e facilitar a ação dos métodos tópicos de tratamento e dos procedimentos cirúrgicos. Nesta fase, o desbridamento cirúrgico dos tecidos necróticos é muito importante e inclui a remoção dos tecidos desvitalizados e/ou infectados do leito da ferida. O papel dos curativos relaciona-se com a absorção do exsudato da ferida e com a manutenção de um meio úmido, que facilita os mecanismos de cicatrização ${ }^{28}$.

Nesse contexto, destaca-se a terapia por pressão negativa (sistema a vácuo), através do uso de esponjas que ficam em contato com o leito da ferida e isoladas do meio por adesivos plásticos, sobre a qual é aplicada uma sucção com pressão
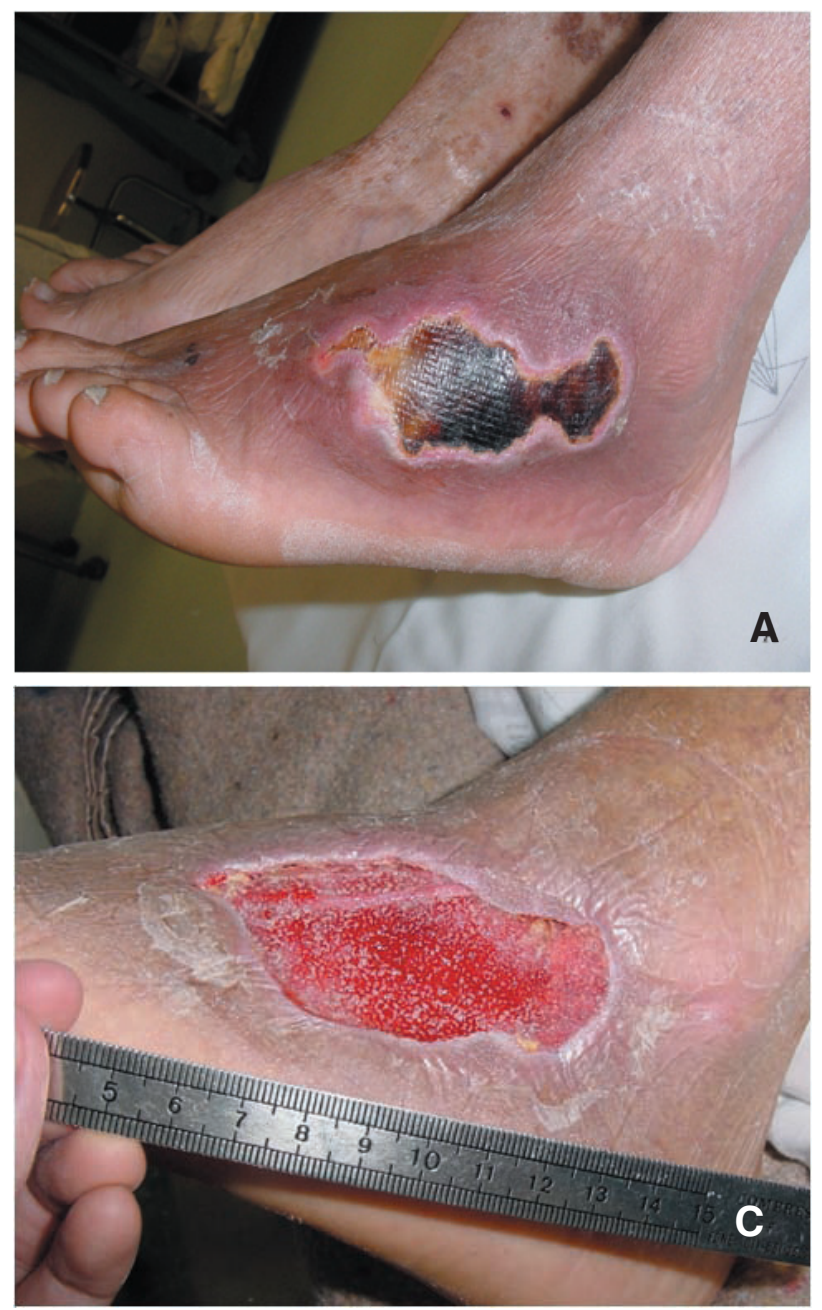

subatmosférica ${ }^{29}$. Esse sistema auxilia na redução do edema, na melhora do aporte sanguíneo ao leito e na formação do tecido de granulação, acelerando a resolução dessas feridas e tornando mais precoce a indicação do tratamento cirúrgico definitivo.

Após o preparo do leito, as feridas diabéticas mais simples podem evoluir para cicatrização. Contudo, as feridas mais complexas demandam a complementação do tratamento cirúrgico através do uso de enxertos de pele, de retalhos locais ou de retalhos microcirúrgicos, com o objetivo de promover a cobertura dos tecidos e a proteção de estruturas nobres como ossos, articulações, tendões, vasos e nervos (Figura 1).
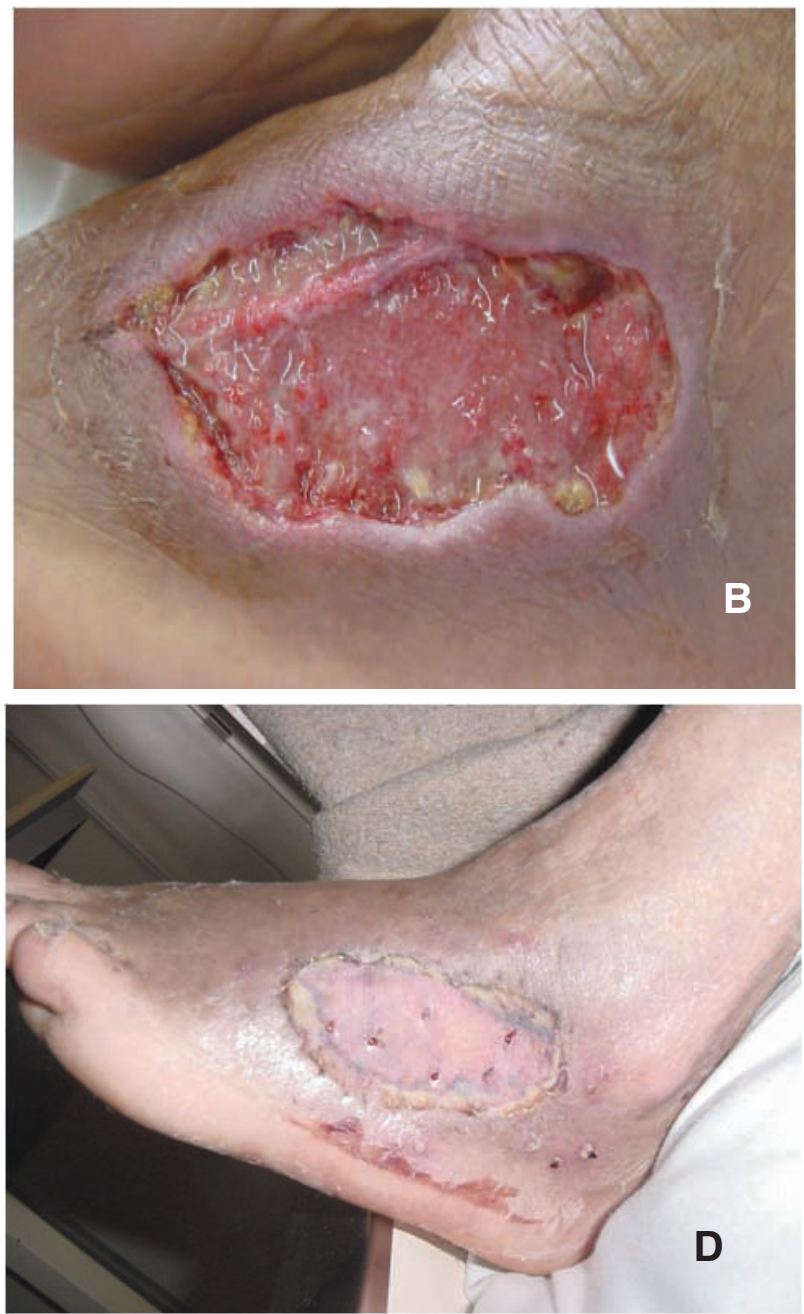

FIGURA 1. Exemplo do protocolo de atendimento às feridas do diabético adotado no Serviço de Cirurgia Plástica do HCFMUSP. Em (A) observa-se a escara, em (B) a lesão logo após a limpeza cirúrgica, em (C) o aspecto do tecido de granulação após remoção do vácuo e em (D) aspecto após cinco semanas da enxertia de pele

O diagnóstico precoce e o tratamento adequado das feridas nos pés de pacientes diabéticos colaboram de forma crucial para evitar complicações locais mais graves, e, em última análise, também ajuda na prevenção das amputações. Para isso, uma equipe multidisciplinar deve estar treinada e organizada para oferecer ao paciente um tratamento completo e efetivo. 
Carvalho VF, Coltro OS, Ferreira MC. Wounds in diabetic patients. Rev Med (São Paulo). 2010 jul.-dez.;89(3/4):164-9.

\begin{abstract}
SUMMARY: Diabetes, in Brasil, affects $10 \%$ of the population over 40 years of age Among the serious complications, those on the lower extremities are the majority (40 to $70 \%$ ). Microangiopathy affects the small vessels the blood supply of nerves, the vasa nervorum and causes the peripheral neuropathy, retinopathy and nephropathy. Neuropathic process enhances osseous deformities on the the foot, which increase plantar pressures in an insensitive foot, ending in ulcerations. Since 1988 the development of the Pressure Specified Sensory Device ${ }^{\mathrm{TM}}$ - PSSD turn out to be a single device able to quantify thresholds of pressure, necessary for the patient to feel one or two points tested, statically and in movement. We can now better access the sensitive deficit on the diabetic patients and plan appropriate prevention measures. Treatment of the diabetic wound involves ischemia control, if necessary and surgical debridement in order to remove necrotic tissues. The new developed vacuum system helps the reduction of edema, enhance blood perfusion to the wound and granulation tissue growth providing faster bed preparation and earlier surgical repair usually with skin grafts.
\end{abstract}

KEY WORDS: Wounds and injuries/complications; Diabetic foot/complications; Desbridement/ complications; Pressure ulcer; Surgery, plastic.

\title{
REFERÊNCIAS
}

1. Klein R. Hyperglycemia and microvascular and macrovascular disease in diabetes. Diabetes Care. 1995;18:258-68.

2. Klein R, Kelin BEK, Moss SE. Relation of glycemic control to diabete microvascular comlications in diabetes mellitus. Ann Intern Med. 1996;124:90-96.

3. Klein R, Kelin BEK, Moss SE, Cruickshanks KJ. Relationship of hyperglicemia to the long-term incidence and progression of diabetic retinophaty. Arch Intern Med. 1994;154:2169-78.

4. Reiber GE, Lipsky BA, Gibbons GW. The burden of diabetic foot ulcer. Am J Surg. 1998;176(Suppl 2a):5-10.

5. Dyck PJ, Karnes JL, O'Brien PC, Litchy WJ, Low PA, Melton LJ 3rd. The Rochester Diabetic Neuropathy Study: reassessment of tests and criteria for diagnosis and staged severity. Neurology. 1992;42:1164-70.

6. DCCT Research Group. The effect of intensive diabetes therapy on the development and progression of neuropathy. Ann Intern Med. 1995;122:561-8.

7. Bridges Jr RM, Deitch EA. Infecções do pé diabético. Fisiopatologia e tratamento. In: Deitch EA. Infecções cirúrgicas. Clínicas cirúrgicas da América do Norte. Rio de Janeiro: Interlivros Edições; 1994. Cap.3, p.581600.

8. Williamson JR, Titlon RG, Chang K, Kilo C. Basement membrane abnormalities in diabetes mellitus: relationship to clinical microangiopathy. Diabetes Metab Rev. 1988;4:339-70.

9. Cohen RA. Disfunction of vascular endothelium in diabetes mellitus. Circulation. 1993;87:67-76.

10. Cameron NE, Cotter MA, Basso M, Hohman TC. Comparison of the effects of inhibitors of aldose reductase and sorbitol dehydrogenase on neurovascular function, nerve conduction and tissue polyol pathway metabolites in streptozotocin diabetic rats. Diabetologia. 1997;40:271-81.

11. Johnson JTH. Neuropathic fractures and joint injuries. J Bone Joint Surg. 1967;49-A:1-30.

12. Kristiansen B. Ankle and foot fractures in diabetics provoking neuropathic joint changes. Acta Orthop Scand. 1980;51:975-9.

13. Reiber GE, Boyko EJ, Smith DG. Lower extremity foot ulcer and amputations in diabetes. In: National Diabetes Data Group, editors. Diabetes in America. 2nd ed. Washington, DC: DHHS; 1995.

14. Moss SE, Klein R, Klein B. The 14-year incidence of lower-extremity amputation in a diabetic population. Diabetes Care. 1999;22:951-9.

15. Ramsey SD, Newton K, Blough D, McCulloch DK, Sandhu NA, Reiber GE, Wagner EH. Incidence, outcomes, and cost of foot ulcers in patients with diabetes from two settings. Diabetes Care. 1999;22:382-7.

16. Rith-Najarian SJ, Stolusky T, Gohdes DM. Identifying diabetic patients at high risk for lower-extremity amputation in a primary health care setting. Diabetes Care. 1992;15:1386-9.

17. Walters DP, Gatling W, Mullee MA, Hill RD. The distribution and severity of diabetic foot disease: a community study with comparison to a non-diabetic group. Diabetes Med. 1992;9:354-8.

18. Boyko E, Ahroni JH, Stensel V, Forsberg RC, Davignon DR, Smith DG. A prospective study of risk factors for diabetic foot ulcer. The Seattle Diabetic Foot Study. Diabetes Care. 1999;22:1036-42. 
19. McNeely MJ, Boyko EJ, Ahroni JH, Stensel VL, Reiber GE, Smith DG, Pecoraro RF. The independent contributions of diabetic neuropathy in foot ulceration. How great are the risks? Diabetes Care. 1995;18:216-9.

20. Abbott CA, Carrington AL, Ashe H, Banheira S, Todo LC, Griffiths J, et al. The North-West foot care study: incidence of, and risks of the diabetic foot ulceration in a community-based patient cohort. Diabetes Med. 2002;5:377-84.

21. Clark CM, Lee A. Prevention and treatment of the complications of diabetes mellitus. New Engl J Med. 1995;332:1210-7.

22. Altman MI, Altman KS. The podiatric assessment of the diabetic lower extremity: special considerations. Wounds. 2000;12(Suppl B):64B-71B.

23. Boike AM, Hall JO. A practical guide for examining and treating the diabetic foot. Cleve Clin J Med. 2002;69:342-8.
24. Singh N, Armstrong DG, Lipsky BA. Preventing foot ulcers in patients with diabetes. JAMA. 2005;293(2):217-28.

25. Omer GE Jr. Methods of assessment of injury and recovery of peripheral nerves. Surg Clin North Am. 1981;61:303-19.

26. Malgrange D, Richard JL, Leymarie F. Screening diabetic patients at risk for foot ulceration. A multicentre hospital-based study in France. Diabetes Metabol. 2003;29(3):261-8.

27. Dellon AL. Clinical use of vibratory stimuli to evaluate peripheral nerve injury and compression neuropathy. Plast Reconstr Surg. 1980;65:466-76.

28. Ferreira MC, Tuma Jr P, Carvalho VF, Kamamoto F. Complex wounds. Clinics. 2006;61:571-8.

29. Ferreira MC, Carvalho VF, Kamamoto F, Tuma P Jr, Paggiaro AO. Negative pressure therapy (vacuum) for wound bed preparation among diabetic patients: case series. Sao Paulo Med J. 2009;127:166-70. 